\title{
ECG study in practical labs for biomedical engineering training
}

\author{
Pablo Pérez García, Alberto Olmo Fernández, Alberto Yúfera García \\ Electronic Technology Department, Higher School of Computer Engineering \\ University of Seville \\ Seville, Spain \\ pablopg@us.es
}

\begin{abstract}
Non-invasive biomedical measurements are one of the most important technological contributions within the biomedical engineering field. On this paper, a dual laboratory session student oriented is designed to simulate and implement a cardiac signal monitor. During the first session, ORCAD PSpice software is used to simulate the whole process. The students can acquire knowledge on the process by configuring and running both the instrumentation amplifier and a passive filter to improve the signal quality. The second session requires from the student basic laboratory skills to use a specific printed circuit board (PCB) to measure its very own cardiac potential. As a result, from this session, the student can visualize the ECG signal acquired directly on the laboratory oscilloscope.
\end{abstract}

Keywords-Biomedical; Instrumentation; Amplifiers; Noninvasive measurement; ECG.

\section{INTRODUCTION}

This work is presented within the Electromedicine course held under the "Health Engineering Degree. Biomedical Engineering mention" in the Higher Technical School of Computer Engineering, University of Seville. The lab sessions for this course aims at introducing the students to the electronic devices being employed on the medical field for healthcare in diagnosis, treatment and research [1].

To accomplish such purpose a project-based learning is proposed during the laboratory sessions. The students face a set of real world electromedicine problems. Of great interest is the analysis of non-invasive biomedical signals like the study case presented in this paper; cardiac potentials. Cardiac potential analysis is a very important medical tool extensively used to detect potential life-threaten conditions on patients worldwide [2]. It is a simple technique consisting on the observation of electrical potentials at the patient skin using appropriate instrumentation. The students achieve a great degree of insight in the measurement of such signal during the laboratory session.

In addition, an important set of skills are empowered during the learning sessions forming this block at this Electromedicine course. The students familiarize themselves with the engineering approach to a real-life problem; step by step. First of all, to develop a certain amount of intuition on the circuit behaviour, they are asked to implement the circuit on a PSpice simulator, for such purpose they are provided with typical sample readings from the skin, and their task is to understand the instrumentation amplifier and obtain the electrocardiogram signal on the simulator which corresponds to that sample input. Secondly, they are asked to implement and test the circuit using a specifically designed printed circuit board (PCB) and a set of electronic components and they will test the instrumentation amplifier to verify its simulated behavior and finally use it along with the PCB to observe their very own electrocardiograms on the oscilloscope.

\section{METHODS AND MATERIALS}

\section{A. Circuit for cardiac potential measurement}

The work presented here illustrates a possible setup for the measurement of biopotential, more specifically, cardiac potentials, using discrete electronic modules [3]. The necessary components are an instrumentation amplifier, the electrodes for the body-amplifier interface and the passive components for perform the filtering before acquiring the signal with the oscilloscope. The basic circuit to achieve such purpose is presented on Figure 1.

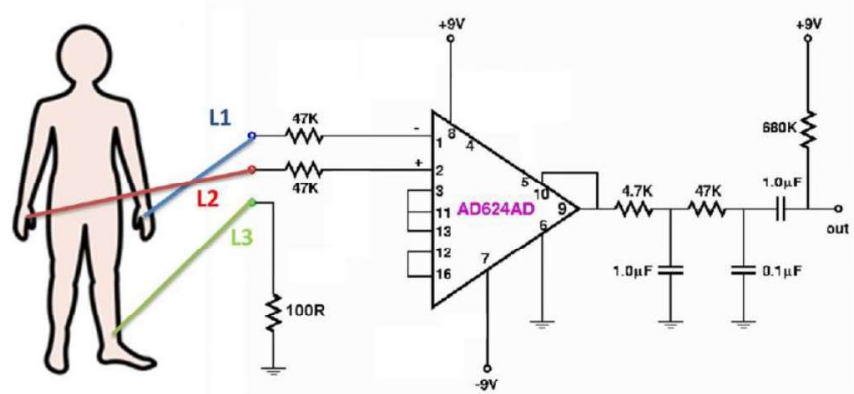

Figure 1: Basic electrocardiography circuit.

\section{B. Materials}

The student is provided with a printed circuit board which was designed for this laboratory sessions by the Electronic Technology department from University of Seville [4]. This board implements all the necessary components for acquiring the signal and it is shown on the figure 2 . The three main connectors correspond to power, signal output and body electrodes, furthermore, the passive components which form 


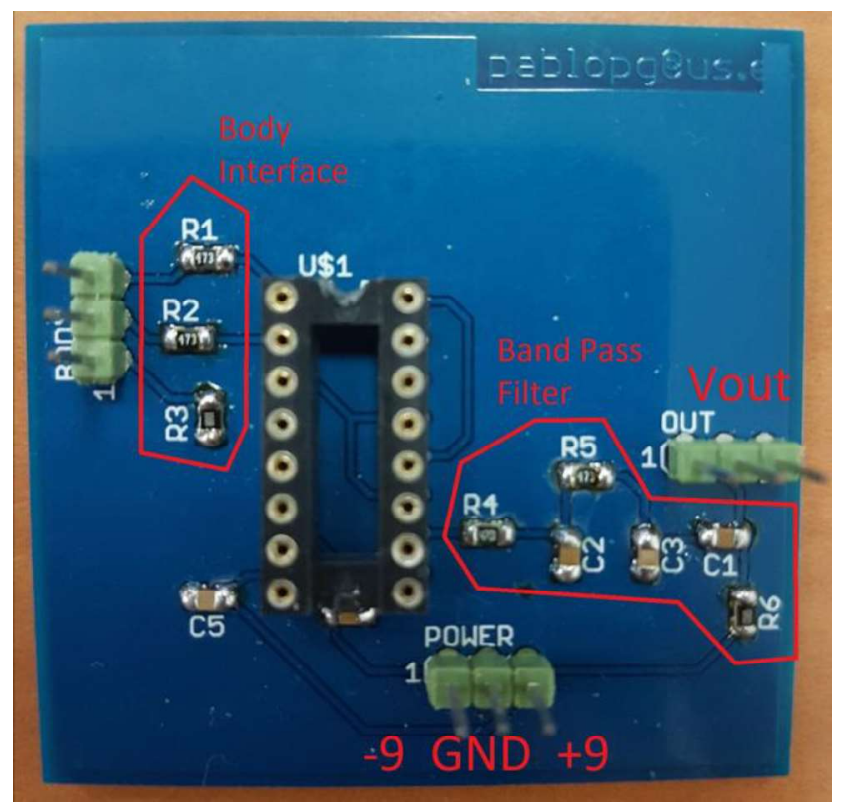

Figure 2: Printed Circuit Board for the test.

the filtering stage are also included setting the system ready for performing the ECG visualization.

The Instrumentation amplifier which fits on the socket in the $\mathrm{PCB}$ is the AD624AD. For performing the cardiac potential acquisition task, it is necessary to configure the pinout amplifier to a gain of around 1000. This is performed by connecting pins 13,11 to pin 3 and pin 12 to 16 . The amplifier features are:

- Low noise: $0.2 \mu \mathrm{Vpp}$.

- High CMRR: $130 \mathrm{~dB}$.

- Low input offset voltage: $25 \mu \mathrm{V}$.

- $\quad$ Pin programmable gains: $1,100,200,500,1000$.

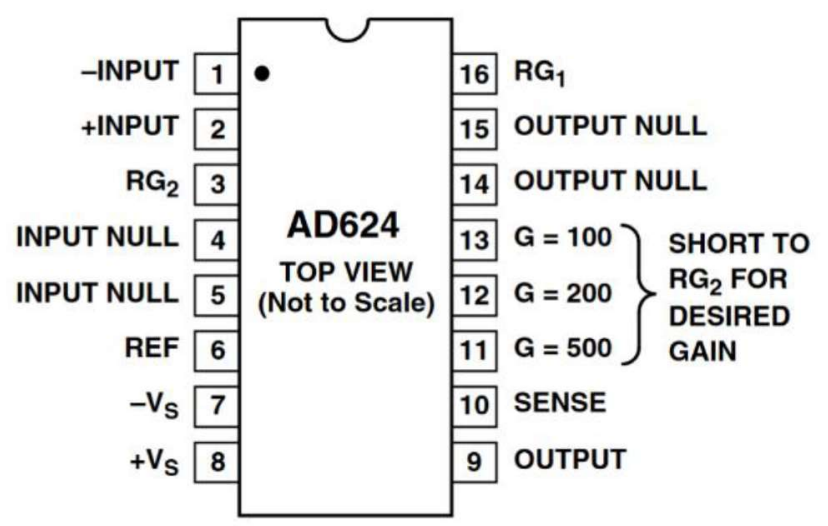

Figure 3: AD624AD Schematic and configuration.

The rest of elements for both sessions are:

- Personal Computer with ORCAD PSpice.
- $\quad$ Skin electrodes [5].

- Prototyping board.

- Passive components (resistors, capacitors).

- Oscilloscope.

- Signal Generator.

- $\quad$ Power Supply Units.

\section{LABORATORY SESSIONS}

\section{A. First Session}

During the first laboratory session the students must understand and simulate the whole circuit to acquire the cardiac signal measurements using ORCAD PSpice. The laboratory session was created using this software, since ORCAD implements an easy to use option to define excitation signals from text document data, to serve as input voltages/current, making possible to use the information contained on medical databases to simulate our practical problem.

The session is divided into a set of different tasks; all students must complete a set of tasks to prepare themselves for the real components on the second session.

- Understand the behaviour of an instrumentation amplifier by simulating a simple circuit and measuring the output/input gain.

- Implement a simple band pass filter to acquire the output signal and perform a frequency analysis to verify its compliance with the biosignal under test. Figure 3.

- Use the input signals provided to implement and simulate the whole system.

The result of the simulation is the visualization of the signal presented on the Figure 5. 'ECG' signal on the lab computer.

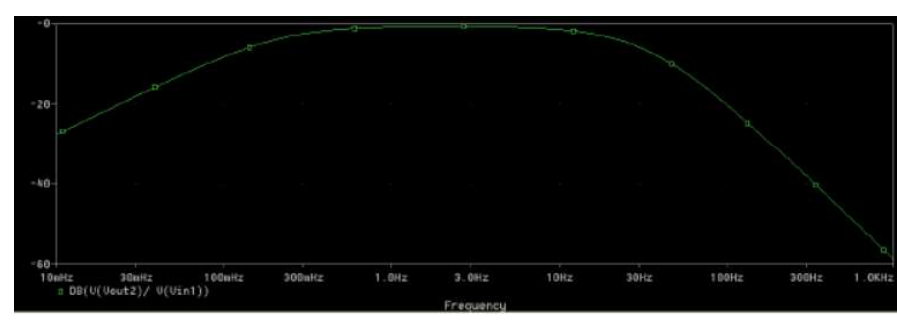

Figure 4: Band Pass Filter Simulation

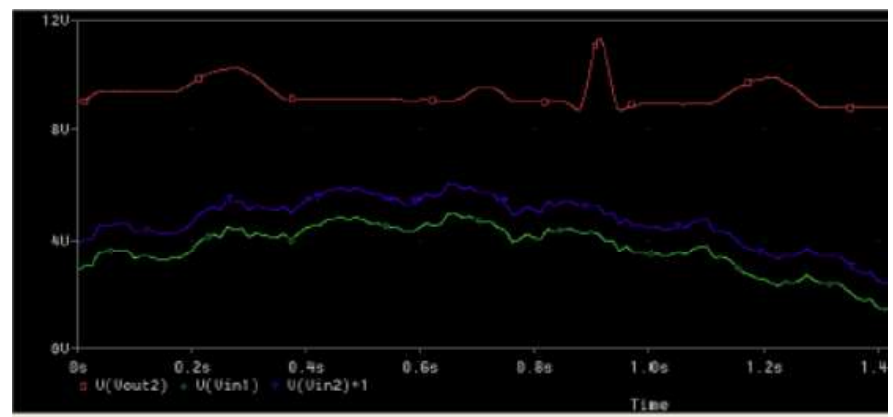

Figure 5: ECG Simulation. Below signals are obtained from input text files and converted to voltage waveforms in PSPICE ORCAD. 


\section{B. Second Session}

On the second session, the work relies on re-implementing the circuits studied on the previous session but using the real components. The first task assigned was to verify the behaviour of the Instrumentation Amplifier in the lab using a signal generator and introducing a small sinusoidal wave $(\sim 5 \mathrm{mV}$ and below) on the differential inputs on the device and coupling the ground references. By configuring adequately, the device the students can observe the output signal as a $5 \mathrm{~V}$ sinusoidal wave, hence verifying the gain value predicted on the simulations.

Once the instrumentation amplifier was tested, next step is to evaluate the small passive band pass filter provided on the small PCB. The students will use now the signal generator to analyse the frequency response and verify the results obtained in the previous lab session, illustrated on Figure 3. They will inject a sinusoidal signal with frequency in the interest range [ ], and measure the output/input amplitude ratio. The signal is applied through the socket and measure the response at the output (figure 2).

The final step is the most expected by the students. They finally mount the instrumentation amplifier on the PCB and evaluate the whole system behaviour. They must power the board and put the electrodes themselves. Sensing electrodes for left hand (L1), right hand (L2) and left foot (L3) are set for each student. After powering up the device and connecting the oscilloscope to the circuit output they are all surprised by the live result from this small circuit. The signal presented on the Figure 5, is an example of the ECG output from this final task. This signal represent the student electrocardiogram amplified a factor of 1000 .

Finally, the students are requested to retrieve the parameters of the signal observed; frequency of beats (RR segment), maximum amplitude along with their physical characteristics (voltage of $\mathrm{R}$ wave); sex and weight, as the report their individual state.

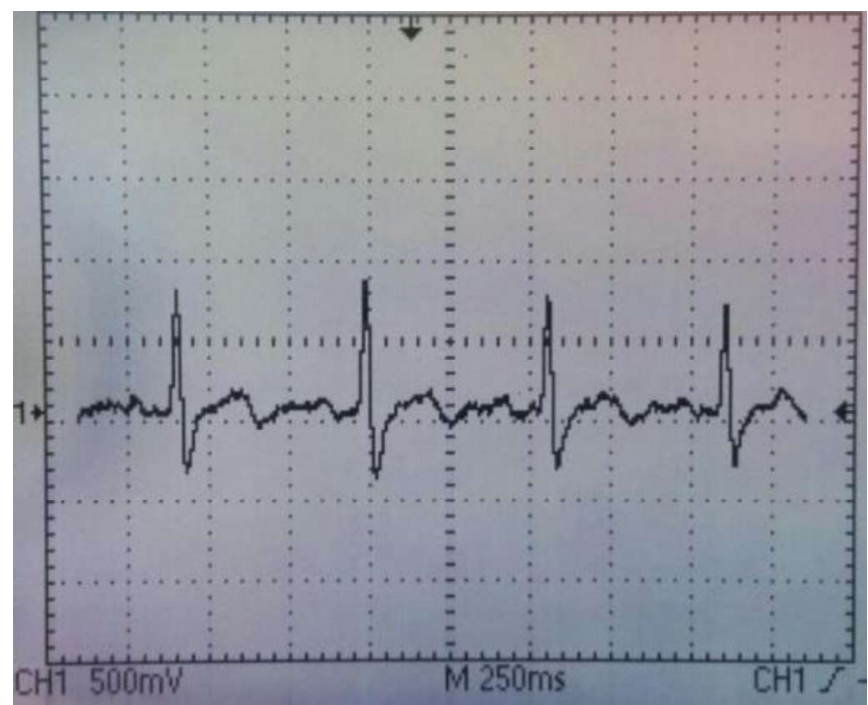

Figure 6: ECG experimentally observed on the oscilloscope by the students.

\section{RESUltS}

\section{A. Student results}

As a final exercise the students were asked to gather some information from the signal displayed on their oscilloscopes, their own ECG. The signal quality was somehow affected by the electrodes connection, and noise level varied intensely among the different students. However, all of them reach the visualization like the depicted on the figure 5 .

Data gathered by the students on the lab is presented on the table 1. The final row on the table is the mean value represented with the standard deviation computed from the experimental data acquired by the students in the laboratory sessions. The final row represents the main value obtained and the standard deviation observed, there are three values considered as outliers since those are clearly out of the main tendency and is probably due to a misread on the oscilloscope scale by a group of students.

Table 1: Results measured by the students.

\begin{tabular}{|c|c|}
\hline Frequency (bpm) & Amplitude (mV) \\
\hline 60 & $2.12 *$ \\
\hline 100 & $2.16^{*}$ \\
\hline 80 & $3.28 *$ \\
\hline 80 & 0.55 \\
\hline 92 & 0.46 \\
\hline 75 & 0.64 \\
\hline 80 & 0.62 \\
\hline 79 & 0.57 \\
\hline 100 & 0.78 \\
\hline 90 & 0.8 \\
\hline 89 & 0.396 \\
\hline 60 & 0.456 \\
\hline 76 & 0.480 \\
\hline 73 & 0.225 \\
\hline 67 & 0.54 \\
\hline 75 & 0.7 \\
\hline 94 & 0.84 \\
\hline 92 & 0.77 \\
\hline 75 & 0.64 \\
\hline 80 & 0.6 \\
\hline 67 & 0.51 \\
\hline 77 & 0.55 \\
\hline 112 & 0.51 \\
\hline 68 & 0.2 \\
\hline 80 & 0.7 \\
\hline 83 & 0.82 \\
\hline 80 & 0.9 \\
\hline 64 & 0.4 \\
\hline 96 & 0.45 \\
\hline 80 & 0.6 \\
\hline 80 & 0.3 \\
\hline 80 & 0.6 \\
\hline $80 \pm 12$ & $0.76 \pm 0.62$ \\
\hline
\end{tabular}


The following graph on Figure 7 depicts the resulting frequency vs amplitude values on a scatter plot style. The tendency confirms that greater bpm involves greater amplitude on the electrical impulse, however the variance is big (same order as the magnitude we are measuring) in the amplitude.

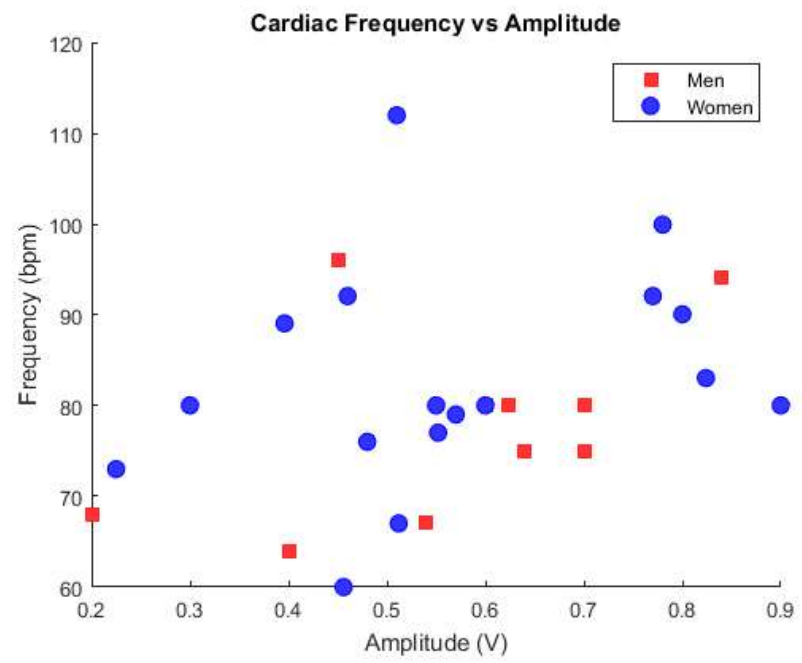

Figure 7: Signal Parameters acquired by the students in the lab.

\section{B. Session outcomes}

From an engineering point of view, it is very important to remark the specific training for the future designers of biomedical instrumentation and devices, in the correct process to create. This session allows simulating, implementing and testing the proposed ECG circuit approach, showing them the importance of achieving a certain intuition into the system under development before actually facing the real circuit.

Additionally, the high level of motivation introduced into most of the students attending the session was a great outcome. They gained an important insight into one of the most employed biomedical non-invasive marker and its acquisition. The electrocardiogram technology illustrated during these sessions has several applications besides monitoring, for instance, smart pacemakers rely on cardiac potential measurements for deciding when the injected pulse will trigger a beat on a patient's heart, thus contributing to power performance and health safety allowing the patients to receive only cardiac stimulation when needed.

For the teachers in the course the most remarkable outcome is the satisfaction and motivation observed on the students which attended the laboratory session. It was considered a great experience and we would like to share the printed circuit board design, it is available on [4].

\section{CONCLUSIONS AND FUTURE WORK}

Besides the strong training achievement and the motivation generated in the students, this work provides a methodology to explore new laboratory session creation, following the same schema.

The course takes part in the fourth year in the Health Engineering degree and it is part of the Biomedical Engineering mention.

During this session the students were able to witness a real medical signal acquisition. They are familiar with courses ranging from physiology and anatomy, to electronics and biomedical instrumentation. This double session improves specific skills on biomedical engineering design using the accumulated knowledge from the syllabus of several courses in the degree. Furthermore, the students are trained using an engineering approach to problem, covering several stages; problem definition, solution simulation to gain a certain degree of intuition on the process and solution testing using real electronic components.

A future lab session for the students within the course is under development and could include the simulation and design of a photoplethysmography sensor for pulse and oxygen blood saturation determination, another very useful widespread non-invasive biomedical measurement.

\section{Acknowledgment (HEADING 5)}

The authors would like to thank the students in the Electromedicine laboratory for their dedication and kind response to the laboratory sessions.

We would also like to thank the department technical support team for helping with the implementation and test of the prototypes.

\section{References}

[1] J. G. Webster and J. W. (John W. Clark, Medical instrumentation: application and design. John Wiley \& Sons, 2010.

[2] "Electrocardiogram: MedlinePlus Medical Encyclopedia." [Online]. Available: https://medlineplus.gov/ency/article/003868.htm. [Accessed: 17-Feb-2018].

[3] "Electrocardiogram (ECG) circuit diagram for use with oscilloscopes." [Online]. Available: https://www.picotech.com/library/applicationnote/electrocardiogram-ecg-circuit-for-use-with-oscilloscopes. [Accessed: 09-Feb-2018].

[4] "pablopgus/ElectrocardiogramBoard: A printed circuit board for visualizing electrocardiogram on the oscilloscope using laboratory equipment and an AD624AD instrumentation amplifier" [Online]. Available: https://github.com/pablopgus/ElectrocardiogramBoard. [Accessed: 20-Feb-2018].

[5] "Electrodo Desechable Foam GS43 (Bolsa 50u) - Ribas Medicina." [Online]. Available: https://www.ribasmedicina.com/producto/electrododesechable-foam-gs43-bolsa-50u/. [Accessed: 20-Feb-2018]. 